\title{
Type la supernovae from exploding oxygen-neon white dwarfs
}

\author{
Kai S. Marquardt ${ }^{1,2}$, Stuart A. Sim ${ }^{3,4}$, Ashley J. Ruiter ${ }^{5,4}$, Ivo R. Seitenzahl ${ }^{5,4,1}$, Sebastian T. Ohlmann ${ }^{1,2}$, \\ Markus Kromer ${ }^{6}$, Rüdiger Pakmor ${ }^{2}$, and Friedrich K. Röpke ${ }^{7,2}$ \\ ${ }^{1}$ Institut für Theoretische Physik und Astrophysik, Universität Würzburg, Campus Hubland Nord, Emil-Fischer-Str. 31, \\ 97074 Würzburg, Germany \\ e-mail: kmarquardt@astro.uni-wuerzburg.de \\ 2 Heidelberger Institut für Theoretische Studien, Schloss-Wolfsbrunnenweg 35, 69118 Heidelberg, Germany \\ 3 School of Mathematics and Physics, Queen's University Belfast University Road Belfast, Northern Ireland BT7 1NN, UK \\ ${ }^{4}$ ARC Centre of Excellence for All-Sky Astrophysics (CAASTRO) \\ 5 Research School of Astronomy and Astrophysics, Mount Stromlo Observatory, Weston Creek, ACT 2611, Australia \\ 6 The Oskar Klein Centre \& Department of Astronomy, Stockholm University, AlbaNova, 10691 Stockholm, Sweden \\ 7 Zentrum für Astronomie der Universität Heidelberg, Institut für Theoretische Astrophysik, Philosophenweg 12, 69120 Heidelberg, \\ Germany
}

Received 28 January 2015 / Accepted 2 May 2015

\section{ABSTRACT}

\begin{abstract}
Context. The progenitor problem of Type Ia supernovae (SNe Ia) is still unsolved. Most of these events are thought to be explosions of carbon-oxygen (CO) white dwarfs (WDs), but for many of the explosion scenarios, particularly those involving the externally triggered detonation of a sub-Chandrasekhar mass WD (sub- $M_{\mathrm{Ch}} \mathrm{WD}$ ), there is also a possibility of having an oxygen-neon (ONe) WD as progenitor.

Aims. We simulate detonations of ONe WDs and calculate synthetic observables from these models. The results are compared with detonations in CO WDs of similar mass and observational data of SNe Ia.

Methods. We perform hydrodynamic explosion simulations of detonations in initially hydrostatic ONe WDs for a range of masses below the Chandrasekhar mass $\left(M_{\mathrm{Ch}}\right)$, followed by detailed nucleosynthetic postprocessing with a 384-isotope nuclear reaction network. The results are used to calculate synthetic spectra and light curves, which are then compared with observations of SNe Ia. We also perform binary evolution calculations to determine the number of SNe Ia involving ONe WDs relative to the number of other promising progenitor channels.

Results. The ejecta structures of our simulated detonations in sub- $M_{\mathrm{Ch}}$ ONe WDs are similar to those from CO WDs. There are, however, small systematic deviations in the mass fractions and the ejecta velocities. These lead to spectral features that are systematically less blueshifted. Nevertheless, the synthetic observables of our ONe WD explosions are similar to those obtained from CO models. Conclusions. Our binary evolution calculations show that a significant fraction (3-10\%) of potential progenitor systems should contain an ONe WD. The comparison of our ONe models with our CO models of comparable mass $\left(\sim 1.2 M_{\odot}\right)$ shows that the less blueshifted spectral features fit the observations better, although they are too bright for normal $\mathrm{SNe}$ Ia.
\end{abstract}

Key words. supernovae: general - nuclear reactions, nucleosynthesis, abundances - hydrodynamics - radiative transfer white dwarfs - stars: evolution

\section{Introduction}

Type Ia supernovae (SNe Ia) are believed to result from thermonuclear explosions of white dwarf (WD) stars (Hoyle \& Fowler 1960) in binary systems. The parameters of the progenitor systems (Wang \& Han 2012), however, and the details of the explosion mechanism (e.g. Hillebrandt \& Niemeyer 2000) remain unclear. Several scenarios hold promise for explaining normal or peculiar SNe Ia (see e.g. Hillebrandt et al. 2013). Typically, the exploding WD in all these scenarios is assumed to be a carbon-oxygen (CO) WD. Here, we explore the possibility of explosions in ONe WDs.

For a long time, explosions of near-Chandrasekhar mass WDs (near- $M_{\mathrm{Ch}}$ WDs) formed in the single-degenerate progenitor channel were the favoured model of SNe Ia (see Hillebrandt \& Niemeyer 2000). In this scenario, ONe WDs are excluded because, although electron captures on ${ }^{24} \mathrm{Mg}$ and ${ }^{20} \mathrm{Ne}$ can ignite a nuclear flame, further electron captures in the O-burning ashes lead to loss of pressure support and collapse (Miyaji et al. 1980; Miyaji \& Nomoto 1987). They are therefore expected to form neutron stars rather than being disrupted in a thermonuclear explosion as their mass approaches $M_{\mathrm{Ch}}$ (Nomoto 1984, 1987; Nomoto \& Kondo 1991). However, there is mounting evidence for other progenitor channels contributing to (or dominating) the sample of SNe Ia (e.g. Stritzinger et al. 2006; Ruiter et al. 2009; Gilfanov \& Bogdán 2010; Sim et al. 2010; Scalzo et al. 2014a,b). Many of these alternatives involve detonations in sub$M_{\mathrm{Ch}}$ WDs. This again raises the question of whether ONe WDs contribute to the progenitor population, since sub- $M_{\mathrm{Ch}}$ configurations are stable against gravitational collapse and detonations propagate rapidly enough such that electron captures do not lead to a collapse. This, however, requires the triggering of a detonation in ONe WD matter, which may be possible but has not yet been proven to work (Shen \& Bildsten 2014). Detonations in ONe WDs could be ignited in the double detonation (Livne 1990; Livne \& Glasner 1990, 1991; Woosley \& Weaver 1994; Fink et al. 2007, 2010; Woosley \& Kasen 2011; Moll \& Woosley 2013) or violent merger (Pakmor et al. 2010, 2012, 2013; Moll et al. 2014) scenarios. Despite the uncertainties related to their ignition, we here investigate the question of how, if they do 
Table 1. Relative rates averaged over a Hubble time for theoretically predicted explosion scenarios that may lead to SNe Ia.

\begin{tabular}{lc}
\hline \hline SN Ia progenitor scenario & Rel. rate \\
\hline CO+CO mergers (all) & 1.0 \\
CO+CO mergers (primary mass $\left.>0.9 M_{\odot}\right)^{*}$ & 0.27 \\
ONe+X mergers (all)* & 0.04 \\
ddet $($ CO primary, all) & 0.86 \\
ddet (CO primary mass $\left.>0.9 M_{\odot}\right)^{*}$ & 0.37 \\
ddet (ONe primary; all)* & 0.03 \\
Chandrasekhar-mass CO WD (SD) & 0.01 \\
accretion-induced collapse in ONe WD (AIC) & 0.02 \\
\hline
\end{tabular}

Notes. Data are extracted from the P-MDS model of Ruiter et al. (2014). All values have been normalized to the number of carbon-oxygen WD mergers (full mass range). The double-detonation systems are denoted by ddet. The accretion induced collapse systems arise from ONe WDs that collapse to a neutron star as they approach $M_{\mathrm{Ch}}$ while accreting stably from a stellar companion. Entries marked with an asterisk are the systems for which we show delay times in Fig. 1.

occur, such events might differ from those with CO WD progenitors and whether they might be identifiable as a subpopulation of SNe Ia.

\section{Population synthesis and the origin of ONe WDs}

Garcia-Berro et al. (1997) and Gil-Pons \& García-Berro (2001) investigated asymptotic giant branch (AGB) stars with zero-age main sequence (ZAMS) masses of 9-10 $M_{\odot}$; the first study is for a single star while the second paper describes the evolution of a close binary system. These stars produce ONe WDs with total masses of about $1.1 M_{\odot}$. They are mainly composed of ${ }^{16} \mathrm{O}$ and ${ }^{20} \mathrm{Ne}$, but they also contain some ${ }^{12} \mathrm{C}$, the exact amount of which depends on the initial model ${ }^{1}$. For a $9 M_{\odot}$ ZAMS progenitor, for instance, the ${ }^{12} \mathrm{C}$ mass fraction in the WD material can be up to $\sim 0.05$, but it varies with radius. This $\mathrm{C}$ admixture is important for the initiation and subsequent propagation of the detonation because it acts as an accelerant for neon burning. However, successful initiation and propagation of the detonation are assumptions in the present work. The viability of these can only be addressed by spatially resolved direct numerical simulations of the hydrodynamics coupled to a full nuclear reaction network, unfortunately still out of reach for full star explosion simulations.

The WDs formed in binary systems cover a wider range of masses due to mass gain/loss from/to the binary companion during stellar evolution. Depending on previous mass transfer episodes, the ONe WD can easily have a mass ranging from $1.08 M_{\odot}$ up to $M_{\mathrm{Ch}}$, while CO WDs can be formed with masses as high as $\sim 1.25 M_{\odot}$ (see Hurley et al. 2000). We use the binary population synthesis code STARTRACK (Belczynski et al. 2002, 2008) to predict the number of potential SN Ia progenitors that involve ONe WDs both for dynamical (mergers) and non-dynamical (classic double detonation) scenarios ${ }^{2}$, and show their delay time distribution (DTD; Fig. 1). The results are summarized in Table 1 . We find that averaged over a Hubble time, the total rate of $\mathrm{CO}+\mathrm{CO}$ mergers is $1.06 \times 10^{-13} M_{\odot}^{-1} \mathrm{yr}^{-1}$,

\footnotetext{
1 In addition to effects discussed here, another factor that influences the final composition of the AGB core is the carbon burning rate (Chen et al. 2014).

2 In the case of classic double detonations, the mass ratio is often sufficiently far from unity to enable stable mass transfer to proceed once the larger WD fills its Roche lobe.
}
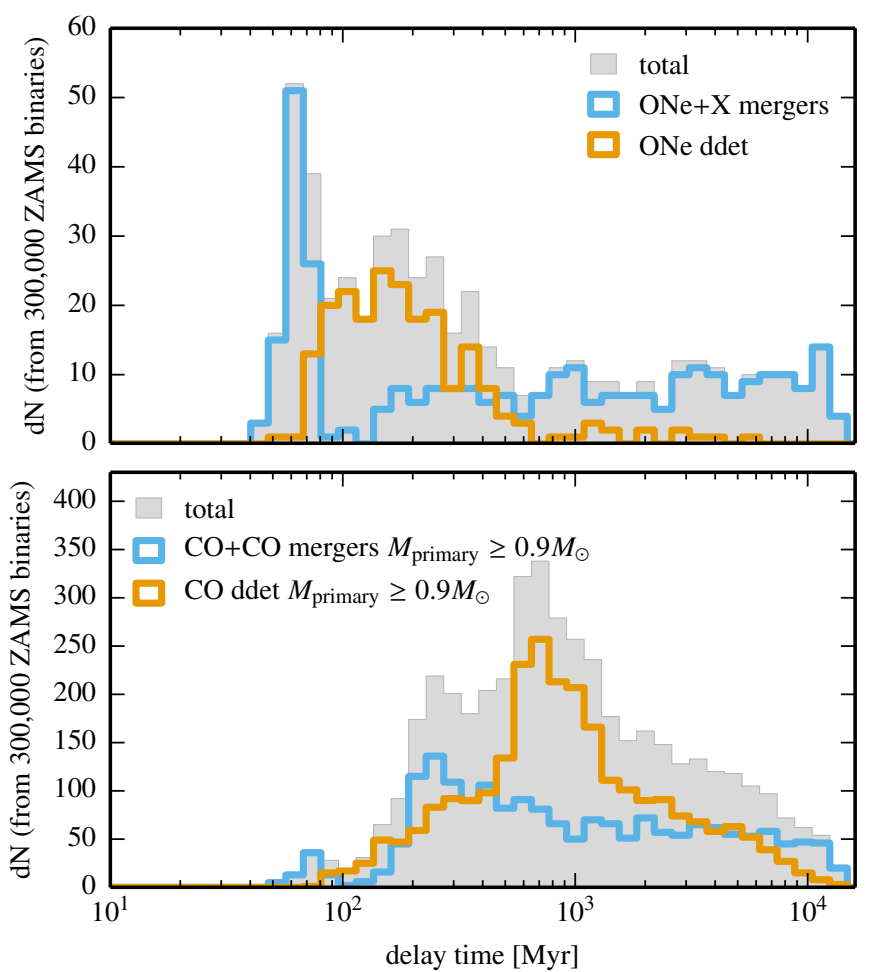

Fig. 1. Delay time distributions for a subset of SN Ia progenitors shown in Table 1. Top panel: mergers that involve one or more ONe WD (blue), and double detonations where the accretor is an ONe WD (orange). Bottom panel: mergers that involve two CO WDs where the primary (more massive) WD is $\geq 0.9 M_{\odot}$ (blue), and double detonations where the accretor is a CO WD with mass $\geq 0.9 M_{\odot}$ (orange). For both panels the combined DTD is shown in grey.

where the mass represents mass born in stars. This value is very close to the estimated SN Ia rate in Milky Way-like galaxies $\left(1.1 \times 10^{-13} M_{\odot}^{-1} \mathrm{yr}^{-1}\right.$, see Badenes \& Maoz 2012). Thus, we use the total number of all $\mathrm{CO}+\mathrm{CO}$ mergers as a reference point when comparing total numbers for various progenitor scenarios in Table 1.

If we compare the systems of $\mathrm{ONe}+\mathrm{ONe}$ WDs combined with the $\mathrm{CO}+\mathrm{ONe}$ WDs (denoted $\mathrm{ONe}+\mathrm{X}$ mergers) that end up in a merger scenario, their fraction is $4 \%$ of the $\mathrm{CO}+\mathrm{CO}$ mergers. Of course, not all $\mathrm{CO}+\mathrm{CO} \mathrm{WD}$ mergers involve primaries massive enough to lead to a bright SN Ia in the violent merger scenario (Pakmor et al. 2010; Ruiter et al. 2013). If we restrict the sample of $\mathrm{CO}+\mathrm{CO}$ mergers to those where the primary WD has at least $0.9 M_{\odot}$, the $\mathrm{ONe}+\mathrm{X}$ merger fraction is as high as $16 \%$. We find that the number of binary systems involving ONe WDs that may lead to classical (non-dynamical mass transfer) doubledetonations are about $8 \%$ of the classical CO double-detonations (see Ruiter et al. 2014). Here, for CO WD primaries, we assume that only the systems in which the primaries are more massive than $0.9 M_{\odot}$ will potentially lead to thermonuclear events that are bright enough to be considered SNe Ia (Sim et al. 2010; Ruiter et al. 2014). The low-mass systems are thus excluded from being considered likely SN Ia candidates; however, we include their numbers in Table 1 for completeness. For the systems involving ONe primaries, the lowest mass primary is $\sim 1.1 M_{\odot}$, thus we include all of them. We note that for the population synthesis model presented, the number of ONe double-detonations is a factor of three higher than single degenerate (SD) systems involving a $M_{\mathrm{Ch}} \mathrm{CO} \mathrm{WD}$. Taken together, these relative rate estimates suggest that potential progenitor systems in which an 
ONe WD explodes are frequent enough to constitute a substantial subset of thermonuclear explosions.

In Fig. 1, top panel, we show the DTDs for double detonations in ONe WDs and double WD mergers involving one or more $\mathrm{ONe}$ WD. The majority of ONe double detonations (orange) have delay times $<650$ Myr. The donor stars in these binaries are naked helium-burning stars that were formed during the second common envelope phase that is encountered during the evolution. A small number of systems with delay times $\gtrsim 1$ Gyr involve helium WD donors. Most of the mergers (blue) are between ONe and CO WDs, though in some cases the mergers are $\mathrm{ONe}+\mathrm{ONe}(14 \%)$. Taking the mergers alone, the DTD shape does not resemble a power law, which has been extensively accredited to merging CO WDs in the literature (see discussion in Totani et al. 2008). In the bottom panel of Fig. 1 we show the DTD for binaries involving CO WDs where the primary CO WD mass for both double detonations and mergers is $\geq 0.9 M_{\odot}$. The double detonations show a very different DTD shape than that of the ONe systems; the peak at $\sim 700 \mathrm{Myr}$ is simply not found in systems with ONe primaries (instead there is a peak $200 \mathrm{Myr}$ ). The lack of a later DTD peak for double detonations involving ONe WDs is due to the fact that events with delay times $\gtrsim 800 \mathrm{Myr}$ typically involve degenerate (helium WD) donors (Ruiter et al. 2014), which are not produced as frequently in binaries involving the more massive $\mathrm{ONe}$ primaries. For mergers involving two CO WDs, again, the DTD shape is quite different in comparison to mergers involving an ONe WD. It is worth noting that the number of ultra-prompt $(<100 \mathrm{Myr})$ mergers in binaries involving an ONe WD is higher than that of $\mathrm{CO}+\mathrm{CO}$ systems, even though the latter (even with the adopted mass cut) outnumber the $\mathrm{ONe}+\mathrm{X}$ mergers by a factor of $\sim 7$. These ultra-prompt events originate from progenitors that undergo two common envelope events where the same star loses its envelope twice (see Ruiter et al. 2013, for discussion). Unlike the mergers with ONe WDs, the $\mathrm{CO}+\mathrm{CO}$ merger DTD peaks around 200-300 Myr, consistent with the previous results of Ruiter et al. (2013). However, cutting out the lower mass CO WD primaries leads to a slightly flatter DTD shape than found when including a larger binary sample, where a $t^{-1}$ power law is typically found.

We do not include mergers between CO WDs and He-rich WDs, which may also lead to double detonations. We find that such systems always have CO WD masses below $0.7 M_{\odot}$ at the time of the merger. Typical masses for systems that lead to unstable mass transfer and merge are $\$ 0.35 M_{\odot}$ and $\sim 0.6 M_{\odot}$ for $\mathrm{He}$ and $\mathrm{CO}$ WDs, respectively. Whether or not a detonation of the $\mathrm{CO}$ core would be triggered by a He-detonation in such lowmass systems is still uncertain (Sim et al. 2012; Shen \& Bildsten 2014), and in fact some of these mergers may lead to the formation of $\mathrm{RCrB}$ stars rather than to thermonuclear explosions (Webbink 1984). Either way, compared to SNe Ia, successful CO ignition in such systems would produce fainter and faster evolving thermonuclear transients (Sim et al. 2012) and would therefore not contribute to the SN Ia rate.

\section{Explosions of ONe white dwarfs}

\subsection{Numerical methods}

For our explosion simulations we use the Eulerian hydrodynamics code LEAFS (Reinecke et al. 1999, 2002; Röpke 2005; Röpke \& Hillebrandt 2005). This finite volume code is based on the PROMETHEUS (Fryxell et al. 1989) implementation of the "Piecewise Parabolic Method" (PPM, Colella \& Woodward 1984). It includes an appropriate equation of state for WD matter based on the equation described by Timmes \& Swesty (2000). The detonation front is modelled using the level set method (Reinecke et al. 1999; Golombek \& Niemeyer 2005; Röpke \& Niemeyer 2007), where the nuclear burning zone is numerically treated as infinitely thin. For numerical efficiency, instead of a full reaction network we use six pseudo-species, ${ }^{12} \mathrm{C},{ }^{16} \mathrm{O},{ }^{20} \mathrm{Ne},{ }^{4} \mathrm{He}$, intermediate mass elements (IME), and iron group elements (IGE), approximately representing fuel and ash compositions. Nuclear statistical equilibrium is followed by a temperature and density dependent mixture of ${ }^{4} \mathrm{He}$ and the IGE species. The ash composition, which depends on fuel density and composition, is read off from tables that are calibrated in a self-consistent manner as described below (Sect. 3.2).

This is sufficient to model the energetics of the detonation process. For convenience, the numerical simulations presented in this work are performed in two dimensions assuming axisymmetry. The numerical resolution is fixed to $1024 \times 1024$ grid cells that co-expand with the explosion of the WD so that all explosion ejecta can be followed to homologous expansion, approximately reached $100 \mathrm{~s}$ after ignition (Röpke 2005). To determine the detailed chemical composition of the ejecta, we apply a nucleosynthetic postprocessing step (Travaglio et al. 2004). It is based on approximately 90000 tracer particles distributed in our 2D hydrodynamical simulation. This is sufficient to obtain converged nucleosynthetic yields (Seitenzahl et al. 2010). These tracer particles are passively advected with the hydrodynamical flow and record the thermodynamic trajectories of representative fluid elements. In the postprocessing we follow the nuclear reactions, using a nuclear reaction network with 384 species (Thielemann et al. 1986, 1990, 1996).

To predict observables from our explosion models we conduct radiative transfer simulations. Because of the spherical symmetry of the ejecta, we map the 2D distribution of the final chemical composition and the ejecta density to a $1 \mathrm{D}$ grid in velocity space, using the same smooth-particle-hydrodynamicslike algorithm that is described in Kromer et al. (2010). The radiative transfer calculations are performed with ARTIS (Sim 2007; Kromer \& Sim 2009). We use the same atomic data as in Gall et al. (2012) For the calculations $1.024 \times 10^{7}$ Monte Carlo packets are used. The radiative transfer calculations start 2 days after explosion and end 120 days after explosion, with the simulation discretized into 111 logarithmically separated time steps.

\subsection{Calibration of the detonation model}

The detonation in our model is not resolved but is represented by a parameterized description. The energy release in the burning process has to be determined and encoded in the fuel and ash composition with the six pseudo-species. To achieve consistent energetics, we employ an iterative calibration procedure (Fink et al. 2010; Ohlmann et al. 2014). The tracer particles are arranged radially in the exploding star with constant distance in $\log \rho$ to ensure resolution of the transition regime between burning to nuclear statistical equilibrium and incomplete Si burning.

In an initial run, complete burning to IGE is assumed everywhere thus releasing the maximum amount of energy. Subsequently, a nucleosynthesis postprocessing step is performed that changes the ash composition in the lower density regions. The result is mapped into the six pseudo-species tabulated as a function of fuel density. This serves as input for the next iteration step. The second parameter of our detonation model is the front propagation speed. It is set to the Chapman Jouguet velocity (CJ), which is determined according to the energy released in each iteration step. For the calculation of the 


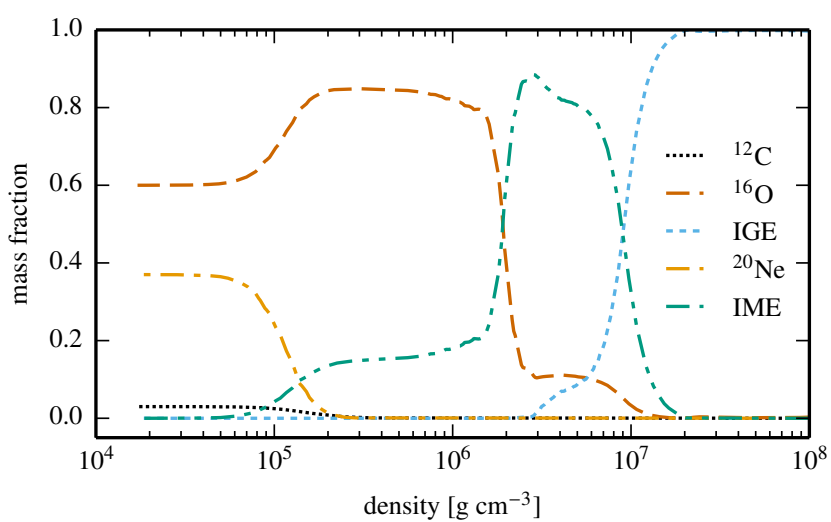

Fig. 2. Illustration of the abundance table for the nuclear burning. The composition of the ash, depending on the density for an initial composition of $X\left({ }^{12} \mathrm{C}\right)=0.03, X\left({ }^{16} \mathrm{O}\right)=0.6$, and $X\left({ }^{20} \mathrm{Ne}\right)=0.37$.

CJ speed, we use the equilibrium sound speed (Fickett \& Davis 1979). The procedure is repeated until the released energy in the explosion run matches the nucleosynthetic postprocessing result which is the case after ten iterations. The values of the calibrated table are plotted in Fig. 2. It clearly shows different burning stages, indicated by the pseudo-species. In the high density regime above $\sim 10^{7} \mathrm{~g} \mathrm{~cm}^{-3}$ fuel material is burned to nuclear statistical equilibrium (NSE) resulting in IGE after freeze-out. For intermediate fuel densities $\left(10^{6} \lesssim \rho\left[\mathrm{g} \mathrm{cm}^{-3}\right] \lesssim 10^{7}\right)$ the ash is composed of IME and oxygen. At lower densities, carbon and neon burn to oxygen, while below $\sim 10^{5} \mathrm{~g} \mathrm{~cm}^{-3}$ burning ceases.

\subsection{Simulation set-up}

We calculate a series of explosion models of ONe WDs. These are set up in hydrostatic equilibrium with central densities $\rho_{0}$ ranging $(1 \ldots 2) \times 10^{8} \mathrm{~g} \mathrm{~cm}^{-3}$, which is well below the threshold for electron captures to become dynamically important (Nomoto 1987 estimates $\rho_{\mathrm{ec}} \simeq 9.5 \times 10^{9} \mathrm{~g} \mathrm{~cm}^{-3}$; and Canal et al. 1992 give $\rho_{\mathrm{ec}} \simeq 8.5 \times 10^{9} \mathrm{~g} \mathrm{~cm}^{-3}$ ). The initial temperature of all models is assumed to be $T=5 \times 10^{5} \mathrm{~K}$ throughout the star. As in our previous work on CO WDs (Sim et al. 2010) we assume uniform composition with values motivated by the results of Garcia-Berro et al. (1997) and Gil-Pons \& García-Berro (2001). Specifically, the mass fractions of our initial composition are $X\left({ }^{12} \mathrm{C}\right)=0.03, X\left({ }^{16} \mathrm{O}\right)=0.6$ and $X\left({ }^{20} \mathrm{Ne}\right)=0.37$. Our set-up procedure results in ONe WDs with masses of $1.18-1.25 M_{\odot}$. Their parameters are summarized in Table 2. Our simulations are for zero-metallicity main-sequence progenitors. Specifically, we do not include any intial abundance of the neutron rich isotope ${ }^{22} \mathrm{Ne}$, which would slightly modify the results (Townsley et al. 2009). As our intention is to study the outcome of detonations in ONe WDs rather than their progenitor evolution and ignition, we ignite the detonation by hand at the centre of the star. For comparison, we also run a simulation of a detonation in a CO WD set up with a central density of $\rho=1.5 \times 10^{8} \mathrm{~g} \mathrm{~cm}^{-3}$, equal mass fractions of carbon and oxygen, and a uniform temperature of $T=5 \times 10^{5} \mathrm{~K}$.

\subsection{Explosion simulations}

From our hydrodynamical simulations we find many similarities, but also some clear differences between the CO WD and the $\mathrm{ONe}$ WD detonations. Table 2 shows the results in terms of kinetic energy of the ejecta and nuclear abundances of the

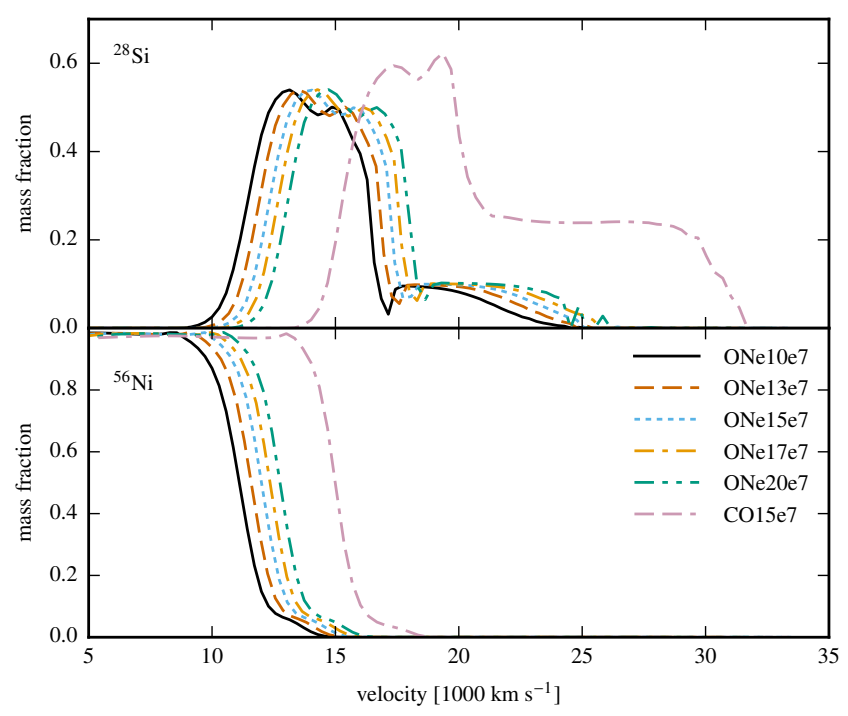

Fig. 3. ${ }^{28} \mathrm{Si}$ (upper panel) and ${ }^{56} \mathrm{Ni}$ (lower panel) mass fractions of the ejecta in velocity space.

pseudo-species. As expected from the differences in the binding energy of the fuel material relative to the ash (the energy release in burning ${ }^{12} \mathrm{C}$ to ${ }^{56} \mathrm{Ni}$ is about $30 \%$ higher than for burning ${ }^{16} \mathrm{O}$ or ${ }^{20} \mathrm{Ne}$ to ${ }^{56} \mathrm{Ni}$; see Wang et al. 2012) the simulations lead to a significantly lower kinetic energy of the ejecta for ONe WD detonations. The nucleosynthetic postprocessing results (Table 2) show that there is a little less ${ }^{56} \mathrm{Ni}$ and overall IGE in the ejecta of the ONe WD detonation than in the ejecta of the CO WD explosion with the same initial mass. In contrast, the IME fraction of the ejecta is somewhat enhanced in the ONe detonation. Both effects are, however, not very pronounced. For the observables, the more important difference is in the distribution of these species in velocity space. This is shown for ${ }^{56} \mathrm{Ni}$ and ${ }^{28} \mathrm{Si}$ as representative examples in Fig. 3. The distribution of both species is shifted towards lower velocities for the ONe WD explosion. Given that the ${ }^{56} \mathrm{Ni}$ masses are similar but the ejecta velocities are lower, we expect that the models will have similar brightness but the ONe WDs will give rise to smaller blueshifts of spectral features. Because of the lower expansion velocities, we expect an increase in the light curve rise time.

\subsection{Comparison of synthetic spectra and observations}

To compare our models with observations we have calculated synthetic spectra and light curves. Table 2 gives important values from these calculations: the time of maximum brightness in $B$ band $\left(t_{B_{\max }}\right)$, the $B$-band decline time scale $\left(\Delta m_{15}(B)\right)$, and the magnitudes at $t_{B_{\max }}$ in the $U, V, R, I, J, H, K$ bands and in bolometric light. Light curves for a subset of our models are shown (and compared to observations) in Fig. 4. There is relatively little variation among the light curves, as one would expect given that the masses of the models are similar. The difference in the velocity structures of the CO WD and the ONe WD detonations lead to a rise time that is approximately two days longer for the latter. The time scales of the infrared light curve evolution are also slower in the ONe models (e.g. the secondary IR peak is reached a little later).

Owing to their large ${ }^{56} \mathrm{Ni}$ masses, our models are too bright to provide a good match to a normal SN Ia such as SN 2005cf (Pastorello et al. 2007; Garavini et al. 2007, Fig. 4). We do, however, find better agreement with the light curves of SN 1991T 
K. S. Marquardt et al.: ONe white dwarfs as SN Ia progenitors

Table 2. Upper half: model parameters and most abundant nuclei of our nuclear network calculations. Lower half: time of $B$-band maximum after explosion, absolute model magnitudes, $\Delta m_{15}(B)$, and wavelength and corresponding blueshift velocity where the absorption of the Si II $6355 \AA$ feature is deepest at $B$-band maximum.

\begin{tabular}{|c|c|c|c|c|c|c|}
\hline Model & ONe10e7 & ONe13e7 & $\mathrm{ONe} 15 \mathrm{e} 7$ & ONe17e7 & ONe20e7 & $\mathrm{CO} 15 \mathrm{e} 7$ \\
\hline$\rho_{0}\left[\mathrm{~g} \mathrm{~cm}^{-3}\right]$ & $1.0 \times 10^{8}$ & $1.3 \times 10^{8}$ & $1.5 \times 10^{8}$ & $1.7 \times 10^{8}$ & $2.0 \times 10^{8}$ & $1.5 \times 10^{8}$ \\
\hline$M_{\mathrm{tot}}\left[M_{\odot}\right]$ & 1.18 & 1.21 & 1.23 & 1.24 & 1.25 & 1.23 \\
\hline$E_{\text {kin }}\left[10^{51} \mathrm{erg}\right]$ & 1.14 & 1.17 & 1.19 & 1.21 & 1.22 & 1.52 \\
\hline $\operatorname{IME}\left[M_{\odot}\right]$ & $2.72 \mathrm{e}-1$ & $2.28 \mathrm{e}-1$ & $2.07 \mathrm{e}-1$ & $1.89 \mathrm{e}-1$ & $1.68 \mathrm{e}-1$ & $1.66 \mathrm{e}-1$ \\
\hline $\operatorname{IGE}\left[M_{\odot}\right]$ & $8.53 \mathrm{e}-1$ & $9.40 \mathrm{e}-1$ & $9.82 \mathrm{e}-1$ & $1.02 \mathrm{e} 0$ & $1.06 \mathrm{e} 0$ & $1.03 \mathrm{e} 0$ \\
\hline${ }^{16} \mathrm{O}\left[M_{\odot}\right]$ & $5.20 \mathrm{e}-2$ & $4.06 \mathrm{e}-2$ & $3.52 \mathrm{e}-2$ & $3.12 \mathrm{e}-2$ & $2.65 \mathrm{e}-2$ & $2.24 \mathrm{e}-2$ \\
\hline${ }^{24} \mathrm{Mg}\left[M_{\odot}\right]$ & $7.21 \mathrm{e}-3$ & $5.72 \mathrm{e}-3$ & $4.98 \mathrm{e}-3$ & $4.41 \mathrm{e}-3$ & $3.76 e-3$ & $6.10 \mathrm{e}-3$ \\
\hline${ }^{28} \mathrm{Si}\left[M_{\odot}\right]$ & $1.38 \mathrm{e}-1$ & $1.16 \mathrm{e}-1$ & $1.05 \mathrm{e}-1$ & $9.57 \mathrm{e}-2$ & $8.47 \mathrm{e}-2$ & $9.30 \mathrm{e}-2$ \\
\hline${ }^{32} \mathrm{~S}\left[M_{\odot}\right]$ & $8.76 \mathrm{e}-2$ & $7.37 \mathrm{e}-2$ & $6.68 \mathrm{e}-2$ & $6.12 \mathrm{e}-2$ & $5.43 \mathrm{e}-2$ & $4.80 \mathrm{e}-2$ \\
\hline${ }^{36} \operatorname{Ar}\left[M_{\odot}\right]$ & $1.95 \mathrm{e}-2$ & $1.65 \mathrm{e}-2$ & $1.51 \mathrm{e}-2$ & $1.38 \mathrm{e}-2$ & $1.24 \mathrm{e}-2$ & $9.91 \mathrm{e}-3$ \\
\hline${ }^{40} \mathrm{Ca}\left[M_{\odot}\right]$ & $1.95 \mathrm{e}-2$ & $1.65 \mathrm{e}-2$ & $1.51 \mathrm{e}-2$ & $1.39 \mathrm{e}-2$ & $1.24 \mathrm{e}-2$ & $9.39 \mathrm{e}-3$ \\
\hline${ }^{52} \mathrm{Fe}\left[M_{\odot}\right]$ & $9.21 \mathrm{e}-3$ & $8.42 \mathrm{e}-3$ & $8.13 e-3$ & $7.95 e-3$ & $7.83 e-3$ & $4.54 \mathrm{e}-3$ \\
\hline${ }^{56} \mathrm{Ni}\left[M_{\odot}\right]$ & $8.32 \mathrm{e}-1$ & $9.16 \mathrm{e}-1$ & $9.57 \mathrm{e}-1$ & $9.90 \mathrm{e}-1$ & $1.03 \mathrm{e} 0$ & $1.00 \mathrm{e} 0$ \\
\hline${ }^{57} \mathrm{Ni}\left[M_{\odot}\right]$ & $6.43 \mathrm{e}-3$ & $7.72 \mathrm{e}-3$ & $8.39 \mathrm{e}-3$ & $8.98 \mathrm{e}-3$ & $9.74 \mathrm{e}-3$ & $1.19 \mathrm{e}-2$ \\
\hline$t_{B_{\max }}$ [days] & 19.1 & 18.9 & 18.7 & 18.4 & 18.4 & 17.3 \\
\hline$M_{B_{\max }}$ & -19.68 & -19.74 & -19.78 & -19.83 & -19.86 & -19.93 \\
\hline$\Delta m_{15}(B)$ & 1.51 & 1.47 & 1.45 & 1.40 & 1.41 & 1.67 \\
\hline$M_{U}\left(t_{B_{\max }}\right)$ & -19.72 & -19.80 & -19.86 & -19.93 & -19.95 & -19.98 \\
\hline$M_{V}\left(t_{B_{\max }}\right)$ & -19.79 & -19.86 & -19.91 & -19.94 & -19.98 & -20.05 \\
\hline$M_{R}\left(t_{B_{\max }}\right)$ & -19.57 & -19.62 & -19.64 & -19.65 & -19.67 & -19.69 \\
\hline$M_{I}\left(t_{B_{\max }}\right)$ & -19.13 & -19.17 & -19.18 & -19.20 & -19.23 & -19.25 \\
\hline$M_{J}\left(t_{B_{\max }}\right)$ & -18.40 & -18.41 & -18.42 & -18.44 & -18.46 & -18.37 \\
\hline$M_{H}\left(t_{B_{\max }}\right)$ & -17.76 & -17.80 & -17.81 & -17.80 & -17.81 & -17.83 \\
\hline$M_{K}\left(t_{B_{\max }}\right)$ & -17.70 & -17.76 & -17.78 & -17.83 & -17.79 & -17.81 \\
\hline$M_{B o l .}\left(t_{B_{\max }}\right)$ & -19.38 & -19.44 & -19.47 & -19.51 & -19.53 & -19.56 \\
\hline$v_{\mathrm{Si} \text { II }}\left[10^{9} \mathrm{~cm} \mathrm{~s}^{-1}\right]$ & 1.3 & 1.3 & 1.4 & 1.4 & 1.4 & 1.7 \\
\hline$\lambda_{\mathrm{Si} \text { II }}[\AA]$ & 6090 & 6080 & 6060 & 6060 & 6060 & 6000 \\
\hline
\end{tabular}

Notes. The first five models are for ONe WDs with a homogeneous composition of $3 \%{ }^{12} \mathrm{C}, 60 \%{ }^{16} \mathrm{O}$, and $37 \%{ }^{20} \mathrm{Ne}$ by mass. The last model, a homogenous $\mathrm{CO}$ WD with equal mass of ${ }^{12} \mathrm{C}$ and ${ }^{16} \mathrm{O}$, is for comparison.

(Filippenko et al. 1992; Phillips et al. 1992; Ruiz-Lapuente et al. 1992; Lira et al. 1998).

In Fig. 5, model spectra are shown at three epochs $(-10,0$, 6 days relative to $\left.t_{B_{\max }}\right)$. Overall, the spectra are similar and compare equally well to the observations; in particular, the important $\mathrm{Si}$ and $\mathrm{S}$ features are clearly present in the models with a strength comparable to the SN 2005cf data. There are, however, differences between the models in detail:

- around maximum light the ONe WD detonation models show slightly stronger IME features;

- the ONe WD explosion models have lower Si velocities (about $\approx 400 \mathrm{~km} \mathrm{~s}^{-1}$; see Table 2), compared to the CO WD explosion model.

These results (see Table 2) confirm what we expect from the hydrodynamical simulations (as discussed above). In all the models the blueshifts (e.g. of the Si II features) are generally too high compared to the observations, but this is less pronounced in the ONe WD detonations (Fig. 5).

The spectral features of our models are much too strong for SN 1991T. This holds in particular for the early epochs $\left(-10 \mathrm{~d}, 0\right.$ days after $\left.t_{B_{\max }}\right)$ where our models show strong Si II and Ca II features, while the spectra of SN 1991T do not show any strong absorption lines. This discrepancy is consistent with expectations based on previous modelling of SN 1991T; in particular, Sasdelli et al. (2014) have shown that the Si in SN $1991 \mathrm{~T}$ should be predominantly located at velocities below $\sim 12500 \mathrm{~km} \mathrm{~s}^{-1}$. In contrast, our models are Si-rich out to $\sim 17000 \mathrm{~km} \mathrm{~s}^{-1}$. Thus, despite having appropriate brightness, the spectra show that our models do not reproduce SN 1991T (or 91T-like objects in general).

\section{Conclusions}

Our population synthesis calculations show that the fraction of potential SN Ia progenitors involving ONe WDs is nonnegligible. For the double-detonation scenario, they account for up to $3 \%$ of the sub- $M_{\mathrm{Ch}}$ systems (see Table 1). For double degenerate mergers, those involving ONe WDs contribute $4 \%$. If we restrict this census to systems that would reach sufficient brightness to produce a SN Ia event, this fraction increases to $11 \%$. In conclusion, explosions of ONe WDs are not a dominant channel of SNe Ia, but our results demonstrate that their contribution is important. It is therefore worthwhile to determine possible outcomes of thermonuclear explosions in ONe WDs in order to establish whether they can be identified as a subpopulation in transient surveys. 


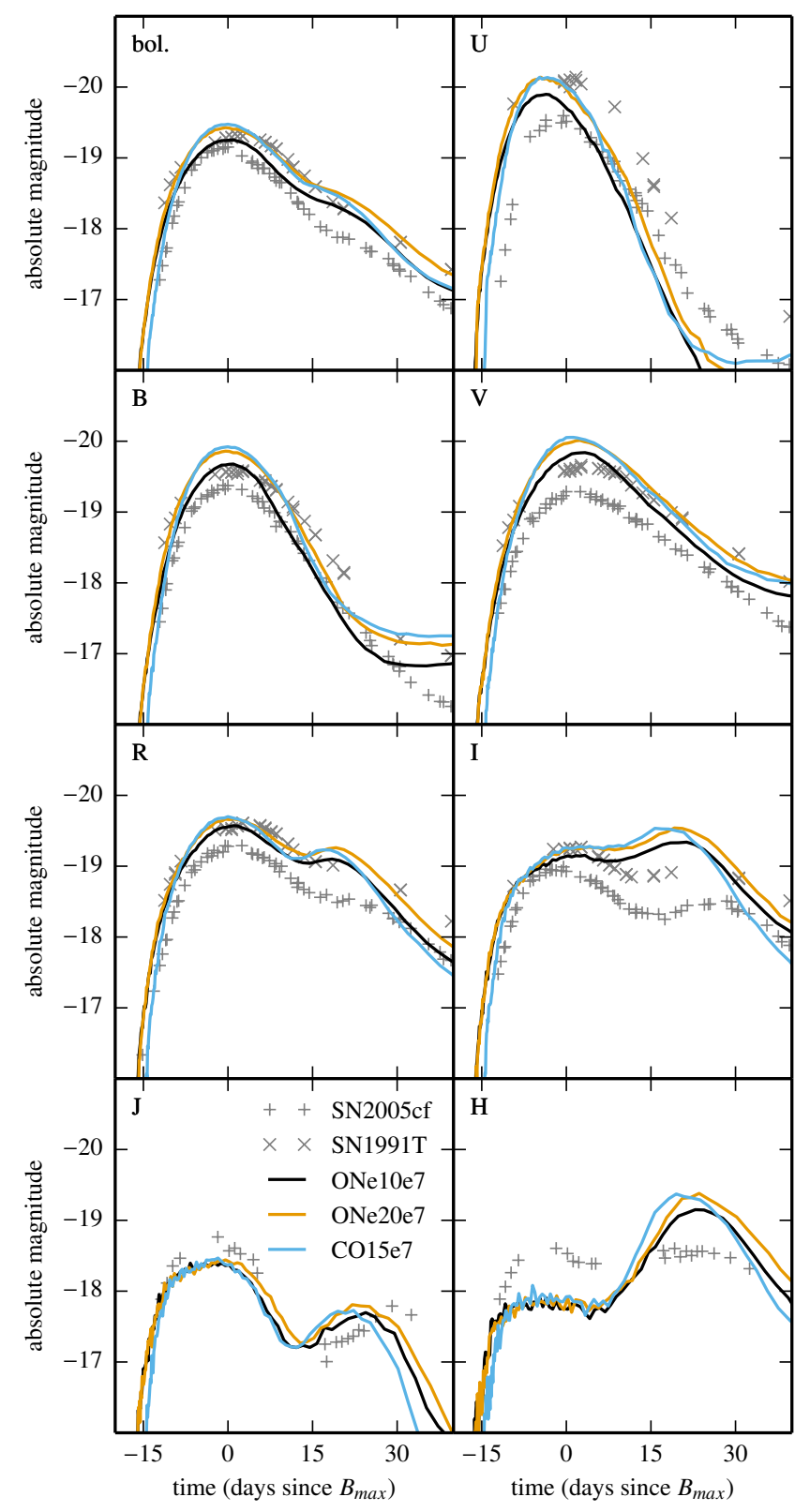

Fig. 4. Synthetic light curves in different bands for our ONe models with a central density of $1 \times 10^{8} \mathrm{~g} \mathrm{~cm}^{-3}$ and $2 \times 10^{8} \mathrm{~g} \mathrm{~cm}^{-3}$ and our CO model compared to the observed light curves of SN 2005cf and $\mathrm{SN}$ 1991T. The SN 2005cf light curve is dereddened for $E(B-V)=$ 0.097 (Pastorello et al. 2007) and the SN 1991T light curve is dereddened using $E(B-V)=0.13$ (Phillips et al. 1992). The distance modules are 30.76 for SN 1991T (Saha \& Thim 2006) and 32.51 for SN 2006cf (Pastorello et al. 2007). For the bolometric light curve of SN 1991T we used a NIR correction based on normal SNe Ia.

We performed simulations of detonations in ONe WDs of different masses and compared these models to detonations in a relatively massive sub- $M_{\mathrm{Ch}} \mathrm{CO}$ WD. As expected, the kinetic energy of the ejecta of ONe WD detonations is lower than that of an equal-mass CO WD. There is, however, very little difference between the composition of the ejecta. Although overall there is no significant improvement or deterioration in matching observed $\mathrm{SNe} \mathrm{Ia}$, the variation in the velocity distribution of species in the ejecta leads to changes in the predicted observables, most importantly in the rise time of the light curves and Si line velocities (both are slower for ONe WD than for

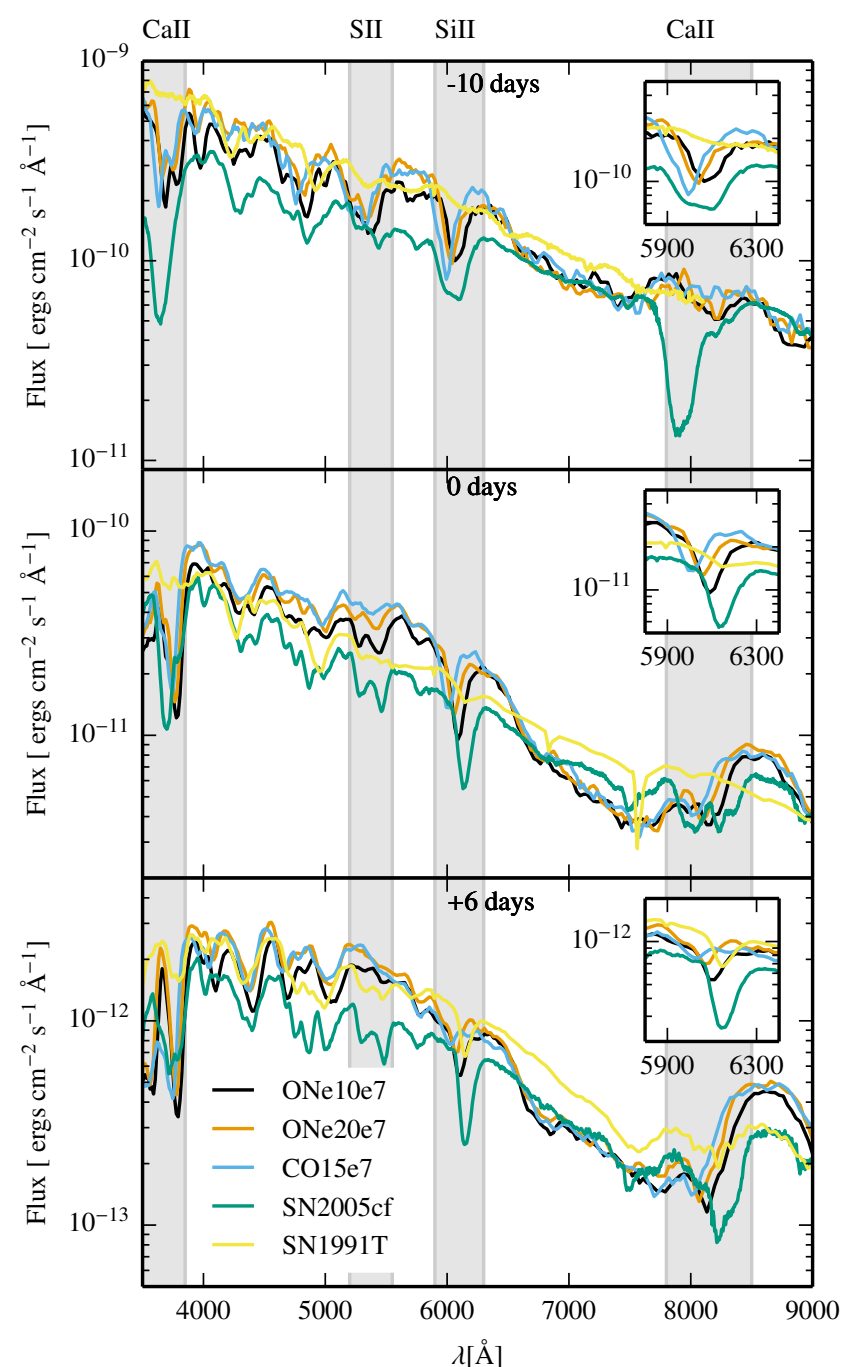

Fig. 5. Synthetic spectra for a subset of our detonation models as outlined by the labels in the bottom panel. For comparison, observed spectra of SN 2005cf $(-10,0,+6 \mathrm{~d})$ and SN 1991T $(-10,-3,+6 \mathrm{~d})$ are shown. All times are relative to maximum brightness in $B$ band. The spectra are de-redshifted: For SN2005cf by $z=0.00646$ and for SN $1991 \mathrm{~T}$ by $z=0.006059$.

CO WD detonations). Thus, with respect to spectral line shifts, our ONe models fit the observations better than our $\mathrm{CO}$ models. In terms of brightness, however, all our models are too bright compared to normal SNe Ia.

The question of whether particular explosion triggering mechanisms can be successful in ONe WDs remains open and has to be addressed in future work.

Acknowledgements. This work was supported by the DFG via the graduate school "Theoretical Astrophysics and Particle Physics" at the University of Würzburg (GRK 1147). Parts of this research were conducted by the Australian Research Council Centre of Excellence for All-sky Astrophysics (CAASTRO) through project number CE110001020 and by the ARC Laureate Grant FL0992131. F.K.R. was supported by the DFG via the Emmy Noether Programme (RO 3676/1-1) and by the ARCHES prize of the German Federal Ministry of Education and Research (BMBF), S.A.S. by STFC grant ST/L000709/1, S.T.O. by the Studienstiftung des deutschen Volkes and R.P. by ERC-StG grant EXAGAL-308037. We also thank the DAAD/Go8 German-Australian exchange programme for travel support and the Partner Time Allocation (Australian National University), the National Computational Merit Allocation and the Flagship Allocation Schemes of the NCI National Facility at the Australian National University. 


\section{References}

Badenes, C., \& Maoz, D. 2012, ApJ, 749, L11

Belczynski, K., Kalogera, V., \& Bulik, T. 2002, ApJ, 572, 407

Belczynski, K., Kalogera, V., Rasio, F. A., et al. 2008, ApJS, 174, 223

Canal, R., Isern, J., \& Labay, J. 1992, ApJ, 398, L49

Chen, M. C., Herwig, F., Denissenkov, P. A., \& Paxton, B. 2014, MNRAS, 440, 1274

Colella, P., \& Woodward, P. R. 1984, J. Comput. Phys., 54, 174

Fickett, W., \& Davis, C. 1979, Detonation, Los Alamos Series in Basic and Applied Sciences, eds. D. H. Sharp, \& L. M. Simmons (University of California Press)

Filippenko, A. V., Richmond, M. W., Matheson, T., et al. 1992, ApJ, 384, L15

Fink, M., Hillebrandt, W., \& Röpke, F. K. 2007, A\&A, 476, 1133

Fink, M., Röpke, F. K., Hillebrandt, W., et al. 2010, A\&A, 514, A53

Fryxell, B. A., Müller, E., \& Arnett, W. D. 1989, Hydrodynamics and nuclear burning, MPA Green Report 449, Max-Planck-Institut für Astrophysik, Garching

Gall, E. E. E., Taubenberger, S., Kromer, M., et al. 2012, MNRAS, 427, 994

Garavini, G., Nobili, S., Taubenberger, S., et al. 2007, A\&A, 471, 527

Garcia-Berro, E., Ritossa, C., \& Iben, I. J. 1997, ApJ, 485, 765

Gil-Pons, P., \& García-Berro, E. 2001, A\&A, 375, 87

Gilfanov, M., \& Bogdán, Á. 2010, Nature, 463, 924

Golombek, I., \& Niemeyer, J. C. 2005, A\&A, 438, 611

Hillebrandt, W., \& Niemeyer, J. C. 2000, ARA\&A, 38, 191

Hillebrandt, W., Kromer, M., Röpke, F. K., \& Ruiter, A. J. 2013, Frontiers of Physics, 8, 116

Hoyle, F., \& Fowler, W. A. 1960, ApJ, 132, 565

Hurley, J. R., Pols, O. R., \& Tout, C. A. 2000, MNRAS, 315, 543

Kromer, M., \& Sim, S. A. 2009, MNRAS, 398, 1809

Kromer, M., Sim, S. A., Fink, M., et al. 2010, ApJ, 719, 1067

Lira, P., Suntzeff, N. B., Phillips, M. M., et al. 1998, AJ, 115, 234

Livne, E. 1990, ApJ, 354, L53

Livne, E., \& Glasner, A. S. 1990, ApJ, 361, 244

Livne, E., \& Glasner, A. S. 1991, ApJ, 370, 272

Miyaji, S., \& Nomoto, K. 1987, ApJ, 318, 307

Miyaji, S., Nomoto, K., Yokoi, K., \& Sugimoto, D. 1980, PASJ, 32, 303

Moll, R., \& Woosley, S. E. 2013, ApJ, 774, 137

Moll, R., Raskin, C., Kasen, D., \& Woosley, S. 2014, ApJ, 785, 105

Nomoto, K. 1984, ApJ, 277, 791

Nomoto, K. 1987, ApJ, 322, 206

Nomoto, K., \& Kondo, Y. 1991, ApJ, 367, L19
Ohlmann, S. T., Kromer, M., Fink, M., et al. 2014, A\&A, 572, A57

Pakmor, R., Kromer, M., Röpke, F. K., et al. 2010, Nature, 463, 61

Pakmor, R., Kromer, M., Taubenberger, S., et al. 2012, ApJ, 747, L10

Pakmor, R., Kromer, M., Taubenberger, S., \& Springel, V. 2013, ApJ, 770, L8

Pastorello, A., Taubenberger, S., Elias-Rosa, N., et al. 2007, MNRAS, 376, 1301

Phillips, M. M., Wells, L. A., Suntzeff, N. B., et al. 1992, AJ, 103, 1632

Reinecke, M., Hillebrandt, W., Niemeyer, J. C., Klein, R., \& Gröbl, A. 1999, A\&A, 347, 724

Reinecke, M., Hillebrandt, W., \& Niemeyer, J. C. 2002, A\&A, 386, 936

Röpke, F. K. 2005, A\&A, 432, 969

Röpke, F. K., \& Hillebrandt, W. 2005, A\&A, 431, 635

Röpke, F. K., \& Niemeyer, J. C. 2007, A\&A, 464, 683

Ruiter, A. J., Belczynski, K., \& Fryer, C. 2009, ApJ, 699, 2026

Ruiter, A. J., Sim, S. A., Pakmor, R., et al. 2013, MNRAS, 429, 1425

Ruiter, A. J., Belczynski, K., Sim, S. A., Seitenzahl, I. R., \& Kwiatkowski, D. 2014, MNRAS, 440, L101

Ruiz-Lapuente, P., Cappellaro, E., Turatto, M., et al. 1992, ApJ, 387, L33

Saha, A., \& Thim, F. 2006, ApJ, 165, 108

Sasdelli, M., Mazzali, P. A., Pian, E., et al. 2014, MNRAS, 445, 711

Scalzo, R., Aldering, G., Antilogus, P., et al. 2014a, MNRAS, 440, 1498

Scalzo, R. A., Ruiter, A. J., \& Sim, S. A. 2014b, MNRAS, 445, 2535

Seitenzahl, I. R., Röpke, F. K., Fink, M., \& Pakmor, R. 2010, MNRAS, 407, 2297

Shen, K. J., \& Bildsten, L. 2014, ApJ, 785, 61

Sim, S. A. 2007, MNRAS, 375, 154

Sim, S. A., Röpke, F. K., Hillebrandt, W., et al. 2010, ApJ, 714, L52

Sim, S. A., Fink, M., Kromer, M., et al. 2012, MNRAS, 420, 3003

Stritzinger, M., Leibundgut, B., Walch, S., \& Contardo, G. 2006, A\&A, 450, 241

Thielemann, F.-K., Nomoto, K., \& Yokoi, K. 1986, A\&A, 158, 17

Thielemann, F.-K., Hashimoto, M.-A., \& Nomoto, K. 1990, ApJ, 349, 222

Thielemann, F.-K., Nomoto, K., \& Hashimoto, M.-A. 1996, ApJ, 460, 408

Timmes, F. X., \& Swesty, F. D. 2000, ApJS, 126, 501

Totani, T., Morokuma, T., Oda, T., Doi, M., \& Yasuda, N. 2008, PASJ, 60, 1327

Townsley, D. M., Jackson, A. P., Calder, A. C., et al. 2009, ApJ, 701, 1582

Travaglio, C., Hillebrandt, W., Reinecke, M., \& Thielemann, F.-K. 2004, A\&A, 425,1029

Wang, B., \& Han, Z. 2012, New Astron. Rev., 56, 122

Wang, M., Audi, G., Wapstra, A., et al. 2012, Chin. Phys. C, 36, 1603

Webbink, R. F. 1984, ApJ, 277, 355

Woosley, S. E., \& Kasen, D. 2011, ApJ, 734, 38

Woosley, S. E., \& Weaver, T. A. 1994, ApJ, 423, 371 